\title{
Endothelial progenitor cell-derived extracellular vesicle-meditated cell-to-cell communication regulates the proliferation and osteoblastic differentiation of bone mesenchymal stromal cells
}

\author{
YUNHAO QIN and CHANGQING ZHANG
}

Department of Orthopaedics, Shanghai Jiaotong University Affiliated Sixth People's Hospital, Shanghai 200030, P.R. China

Received August 5, 2016; Accepted July 13, 2017

DOI: $10.3892 / \mathrm{mmr} .2017 .7403$

\begin{abstract}
Bone tissue engineering is a promising treatment strategy to increase bone regeneration. Endothelial progenitor cells (EPCs) and bone marrow stromal cells (BMSCs) are commonly used to promote vessel formation and osteoblastic differentiation in tissue engineering. Previous studies have demonstrated that EPCs regulate both proliferation and differentiation of BMSCs. However, the underlying mechanism remains unclear. Understanding this mechanism is critical to developing more effective treatments. The role of extracellular vesicles in cell-to-cell communication has attracted substantial attention. These small vesicles deliver proteins, DNA, and RNA and consequently regulate the commitment, function, and differentiation of target cells. In the present study, EPC-derived extracellular vesicles (EPC-EVs were isolated using gradient ultracentrifugation and ultrafiltration and the influence of EPC-EVs on BMSC osteoblastic differentiation and proliferation was examined in vitro. The results indicated that EPC-EVs regulate the osteoblastic differentiation of BMSCs by inhibiting the expression of osteogenic genes and increasing proliferation in vitro. It is suggested that the results regarding the role of EPC-EVs will provide a novel way to explain the crosstalk between EPCs and BMSCs.
\end{abstract}

\section{Introduction}

Bone mesenchymal stromal cells (BMSCs) are plastic adherent cells and are characterized by their multi-potency, and the ability to give rise to colony forming unit-fibroblasts (CFU-F) (1). These types of cells can differentiate into osteogenic, adipogenic and chondrogenic lineages. Therefore, BMSCs are commonly used to promote tissue regeneration.

Correspondence to: Dr Changqing Zhang, Department of Orthopaedics, Shanghai Jiaotong University Affiliated Sixth People's Hospital, 600 Yishan Road, Shanghai 200030, P.R. China E-mail: zhangcq@sjtu.edu.cn

Key words: extracellular vesicles, endothelial progenitor cells, bone mesenchymal stromal cells, osteoblastic differentiation
Bone regeneration is a complicated and highly regulated process. Bones are regenerated in two distinct ways: Intramembranous ossification and endochondral ossification (2). Flat bones arise from intramembranous ossification, and long bones arise from endochondral ossification, and the mesenchyme condensation marks the beginning of both bone regeneration processes (3). In intramembranous ossification, bones are directly formed by osteoblastic differentiation of BMSCs, whereas endochondral ossification involves the cooperation of multiple cell types, during which chondrocytes mediate the growth and formation of the skeleton (4). In endochondral ossification, chondrocytes in the cartilage stop proliferating, undergo hypertrophy, and secrete type $\mathrm{X}$ collagen (5). Hypertrophic chondrocytes guide vessel penetration, osteoblastic differentiation of BMSCs and osteoblast migration. Finally, hypertrophic chondrocytes are programmed to undergo apoptosis, and blood vessels and osteoblasts infiltrate the cartilage matrix and subsequently achieve bone growth and regeneration. Therefore, stimulation of vessel formation and osteoblastic differentiation greatly promote bone regeneration (6).

Bone tissue engineering is a promising therapy to increase vessel formation and bone regeneration (6). An active blood vessel network is a precondition for implants to integrate with the local tissue (7). Endothelial progenitor cells (EPCs) are hematologic precursor cells, defined by Asahara et al (8), that are known for their pro-angiogenic ability (9). The high mobility of EPCs enables the cells to migrate to trauma sites and stimulate neovascularization (10). Therefore, EPCs are often attached to implants to increase vessel penetration. However, the effects of EPCs remain controversial. Duttenhoefer et al (11) identified that EPCs may negatively regulate osteoblastic differentiation of BMSCs in vitro. In contrast, Goerke et al (12) have argued that EPCs support bone regeneration by stimulating vessel formation. Therefore, understanding the communication between EPCs and BMSCs will aid the development of future tissue engineering treatments.

Extracellular vesicles (EVs) are a group of vesicles that include apoptotic bodies, exosomes and microvesicles (13), which are released by almost all cells in the body, including reticulocytes (14), dendritic cells (15), B cells (16), tumor cells (17), mast cells (18), T cells (19), epithelial cells (20) and endothelial cells (21). EVs are widely distributed throughout the body (22) and serve crucial roles in cell-to-cell 
communication. These vesicles can be internalized by the recipient cells through endocytosis (23), and then release functional proteins, DNA and RNA (24). Via this method, EVs can effectively transport and deliver content between cells and regulate homeostasis.

In the present study, the role of EPC-derived EVs in the regulation of osteoblastic differentiation and proliferation of BMSCs was examined. The results demonstrated that EPC-derived EVs can enter BMSCs through endocytosis and release their cargo in the Golgi apparatus, thereby modulating differentiation and proliferation of BMSCs in vitro.

\section{Materials and methods}

Cell culture. Mouse BMSCs were isolated from 7-8-week-old male C57BL/6 mice ( $\mathrm{n}=3$, weight: $20 \mathrm{~g}, 12$-h light/dark cycle, free access to food and water) supplied by the Shanghai Jiaotong University Affiliated Sixth People's Hospital and housed at $22^{\circ} \mathrm{C}$ in $50 \%$ humidity according to established protocols. The use of all samples was approved by and was conducted in accordance with the Ethical Committee of Shanghai Jiaotong University Affiliated Sixth People's Hospital (Shanghai, China). Briefly, BMSCs were collected according to current protocols (25). Cells from passages 3-5 were used in the experiments. BMSCs were cultured with Dulbecco's modified Eagle's medium (DMEM; cat no. 12571071) and 10\% foetal bovine serum (cat no. 10099141) (both from Gibco; Thermo Fisher Scientific, Inc., Waltham, MA, USA) at $37^{\circ} \mathrm{C}$ in a humidified environment with $5 \% \mathrm{CO}_{2}$.

EPCs isolated from the bone marrow of 7-8-week-old C57BL/6 mice were obtained from BioChain (cat no. 7030031). These cells express various endothelial markers, including CD31, CD105, vascular endothelial growth factor receptor 1 and neuropilin-1, and have the spindle morphology common to EPCs. These cells are considered to be late-stage EPCs due to the fact that they are negative for CD133, an early stage endothelial progenitor marker. All EPCs were cultured with DMEM and $10 \%$ foetal bovine serum at $37^{\circ} \mathrm{C}$ in a humidified environment with $5 \% \mathrm{CO}_{2}$. The conditioned medium (EPC-CM) was collected following incubation for $24 \mathrm{~h}$.

For the CFU-F assay, BMSCs were continuously treated with EPC-CM, EPC-derived extracellular vesicles $(5 \mu \mathrm{g} / \mathrm{ml}$ in all experiments, EPC-EV), EV-depleted EPC-CM (noEV) and BMSC culture medium (BMSC-group).

For the osteogenic differentiation assay, the osteogenic medium (OM) contained $0.05 \mathrm{mM}$ ascorbate- 2 phosphate, $10^{-8}$ dexamethasone, and $10 \mathrm{mM} \beta$-glycerophosphate. BMSCs were divided into the following 4 groups: EPC-CM + OM, EPC-EV $+\mathrm{OM}$ group, noEV + OM and the OM group.

Extracellular vesicle isolation. EVs were isolated from the supernatant of EPC-CM following culturing for $24 \mathrm{~h}$ according to current ultracentrifugation protocols (26). Briefly, the EPC culture medium was collected, centrifuged at $500 \mathrm{x} \mathrm{g}$ for $30 \mathrm{~min}$ at room temperature to remove dead cells and then at $16,500 \times \mathrm{g}$ for $20 \mathrm{~min}$ at $4^{\circ} \mathrm{C}$; this was followed by filtration through a $0.22 \mu \mathrm{m}$ filter to eliminate cell debris. Then, EVs underwent ultracentrifugation (Beckman Ti70 rotor; Beckman Coulter, Inc., Brea, CA, USA) at 120,000 x g for $2 \mathrm{~h}$ at $4^{\circ} \mathrm{C}$. The protein content of EVs was measured using a BCA Protein Assay kit (Pierce Biotechnology, Inc., Rockford, IL, USA).

Electron microscopy. Collected EVs were fixed in $4 \%$ paraformaldehyde (PFA) in PBS for $30 \mathrm{~min}$ at room temperature. Fixed EVs were placed onto a formvar carbon-coated grid and dried at room temperature for $20 \mathrm{~min}$. Subsequent to being washed with PBS, the EVs were fixed in $1 \%$ glutaraldehyde for $5 \mathrm{~min}$, washed with water and stained with saturated aqueous uranyl oxalate for $5 \mathrm{~min}$ at room temperature. EVs were then embedded in $0.4 \%$ uranyl acetate and $1.8 \%$ methylcellulose and incubated on ice for $10 \mathrm{~min}$. The excess liquid was then removed. The grid was dried at room temperature for $10 \mathrm{~min}$ and viewed at a magnification of x20,000 using an electron microscope (Philips CM 120; Medical Systems B.V., Eindhoven, The Netherlands).

Confocal microscopy. Harvested EVs were labelled with PKH67 Green Fluorescent Cell Linker (cat no. PKH67GL-1KT; Sigma-Aldrich; Merck KGaA, Darmstadt, Germany) according to the manufacturer's protocols and were then added to BMSCs in the culture medium. A total of $1 \times 10^{5}$ BMSCs were seeded in each well of a 6-well plate. Subsequently, BMSCs were treated with EPC-derived EVs $(5 \mu \mathrm{g} / \mathrm{ml})$ when the cells reached $70-80 \%$ cell confluency. Following $4 \mathrm{~h}$ culture, the cells were washed three times with PBS and then fixed with 4\% PFA. The endoplasmic reticulum (ER-Tracker Red; cat no. E34250), Golgi apparatus (Golgi-RFP; cat no. C10593) and lysosomes (Lyso-Tracker Red; cat no. L12492) (all from Life Technologies; Thermo Fisher Scientific, Inc.) were stained according to the manufacturer's protocols. The cell nuclei were stained with DAPI according to manufacturer's protocol (cat no. BD5010; Bioworld Technology, Inc., St. Louis Park, MN, USA).

CFU-F assay. BMSCs were diluted in DMEM with 10\% FBS and seeded into a 6 -well plate at 100 cells/well and treated with EPC-CM (EPC-CM group) and BMSC culture medium (BMSC). Following 14 days of culture at $37^{\circ} \mathrm{C}$ in a humidified environment with $5 \% \mathrm{CO}_{2}$, the cells were washed three times with 1 X PBS and fixed by addition of ice-cold $100 \%$ ethanol. The cells were stained with $0.1 \%$ crystal violet solution for $10 \mathrm{~min}$ at room temperature. Images were captured by light microscopy.

Alizarin Red staining and MTT. Cells were fixed in $70 \%$ ethanol for $30 \mathrm{~min}$ and rinsed with double-distilled $\mathrm{H}_{2} \mathrm{O}$ and then stained with 40 mM Alizarin Red S (cat no. 130223; Sigma-Aldrich; Merck KGaA), $\mathrm{pH} 4.0$, for 15 min with gentle agitation. Cells were rinsed 3 times with double-distilled $\mathrm{H}_{2} \mathrm{O}$ and then rinsed for 15 min with $1 \mathrm{X}$ PBS with gentle agitation. Images were captured using a light microscope. The MTT (Molecular Probes Life Technologies; Thermo Fisher Scientific, Inc.) assay was performed according to the manufacturer's protocols, and the absorbance was measured at $450 \mathrm{~nm}$ with a microplate reader (MK3; Thermo Fisher Scientific, Inc.).

Western blotting. The cells were washed three times with PBS and lysed in ice-cold lysis buffer [50 mM Tris, pH 7.5, $150 \mathrm{mM}$ 

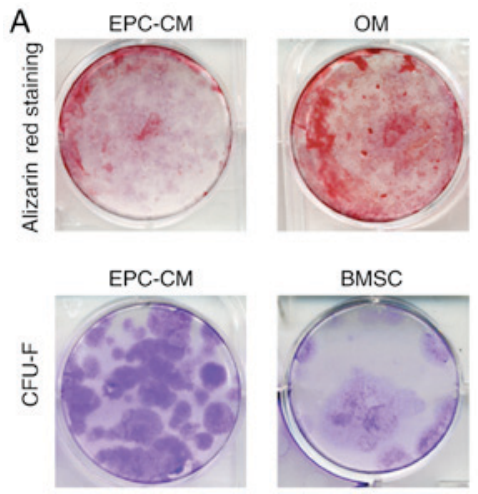

B
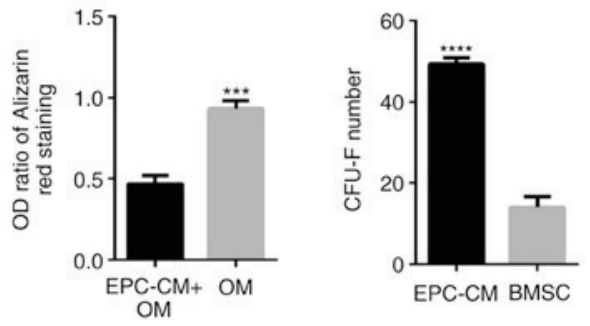

Figure 1. (A) Alizarin Red staining and CFU-F assay for 14 days. (B) The OM group (0.93 \pm 0.02$)$ exhibited a significant increase in calcium deposition, as compared with the EPC-CM + OM group $(0.47 \pm 0.03)(\mathrm{P}<0.05, \mathrm{P}=0.0003)$. There was an increased number of CFU-Fs in the EPC-CM group (49 \pm 3.1$)$ when compared with the BMSC medium group $(14 \pm 1.2 ; \mathrm{P}<0.05, \mathrm{P}=0.000027){ }^{* * * *} \mathrm{P}<0.001$ and ${ }^{* * * *} \mathrm{P}<0.0001$. CFU-F, colony forming unit-fibroblasts; OM, osteogenic medium; EPC, endothelial progenitor cell; CM, conditioned medium; BMSC, bone marrow stromal cell.

$\mathrm{NaCl}, 1 \%(\mathrm{w} / \mathrm{v})$ Nonidet P-40, $0.1 \%(\mathrm{w} / \mathrm{v})$ SDS, and $1 \%(\mathrm{w} / \mathrm{v})$ sodium deoxycholate] supplemented with phenylmethylsulfonyl fluoride (Shen Neng Bo Cai Corporation, Shanghai, China). The lysates were incubated on ice for $30 \mathrm{~min}$ and then centrifuged at $9,000 \times \mathrm{g}$ for $10 \mathrm{~min}$ at $4^{\circ} \mathrm{C}$ to precipitate the debris. The protein concentrations were determined using a BCA protein assay kit (Thermo Fisher Scientific, Inc.). Then, $30 \mu \mathrm{g}$ of the protein lysates were separated using 10 and $18 \%$ (w/v) SDS-polyacrylamide gel electrophoresis (PAGE) and electroblotted onto PVDF membranes (Roche Diagnostics, Basel, Switzerland). Membranes were blocked with 5\% non-fat dry milk in Tris-buffer saline with Tween-20 (TBST) for $2 \mathrm{~h}$ and probed with primary antibodies diluted in TBST containing $5 \%$ milk at $4^{\circ} \mathrm{C}$ overnight. Primary antibodies used included rabbit anti-mouse alkaline phosphatase (ALP) antibodies (cat no. ab108337; 1:1,000), rabbit anti-mouse osteocalcin (OCN) antibodies (cat no. ab93876; 1:1,000), rabbit anti-mouse osteopontin (OPN) antibodies (cat no. ab8448; 1:500), rabbit anti-mouse runt-related transcription factor-2 (RUNX-2) antibodies (cat no. ab23981; 1:1,000) and rabbit anti-mouse GAPDH (cat no. ab9485; 1:1,000) (all from Abcam, Cambridge, MA, USA). Membranes were washed and incubated with horseradish peroxidase conjugated goat anti-rabbit secondary antibody (cat no. A0208; 1:1,000; Beyotime Institute of Biotechnology, Haimen, China) for $1 \mathrm{~h}$ at room temperature. Targeted proteins were visualized using an enhanced chemiluminescence (ECL) detection system (ChemiDoc ${ }^{\mathrm{TM}}$ XRS+ imaging system; Bio-Rad Laboratories, Inc., Hercules, CA, USA).

Reverse transcription-quantitative polymerase chain reaction $(R T-q P C R)$. Osteogenic gene expression in BMSCs at day 14 was measured using RT-qPCR for the following marker genes: ALP, RUNX2, OPN and OCN in BMSCs. Total RNA was extracted using the TRIzol reagent (Thermo Fisher Scientific, Inc.) and qualified by absorbance at $260 \mathrm{~nm}$. cDNA was synthesized from $1 \mu \mathrm{g}$ of RNA by Takara RNA PCR kit (Takara Bio, Inc., Otsu, Japan) following the manufacturer's protocols. Real-time PCR was performed using a SYBR Green Master Mix (Takara, Bio, Inc.) on ABI StepOnePlus system (Applied Biosystems; Thermo Fisher Scientific, Inc.): SYBR Premix Ex Taq, $12.5 \mu \mathrm{l}$; PCR forward primer, $1 \mu \mathrm{l}$; PCR reverse primer $1 \mu \mathrm{l}$; diethylpyrocarbonate water, $8.5 \mu \mathrm{l}$; DNA, $2 \mu 1$. The cycling conditions were: $95^{\circ} \mathrm{C}$ for $30 \mathrm{sec}(1 \mathrm{cycle}) ; 95^{\circ} \mathrm{C}$ for $5 \mathrm{sec}$, then $60^{\circ} \mathrm{C}$ for $30 \mathrm{sec}(40$ cycles). Primer sequences used were: ALP forward, GGC AGCTTGACCTCCTCGGAAGACA and reverse, AGCATG GGGGCCAGACCAAAGATAG; RUNX2 forward, CCC CTCCTACCTGAGCCAGATGACG and reverse, AAGGGC CCAGTTCTGAAGCACCTGA; OPN forward, ACAGCA TCGTCGGGACCAGACTCGT and reverse, GGTAGTGAG TTTTCCTTGGTCGGCG; OCN forward, GCCCTCACA CTCCTCGCCCTATT and reverse, GGGTCTCTTCACTAC CTCGCTGCC; $\beta$-actin forward, CGGGAAATCGTGCGT GACAT and reverse, GGACTCGTCATACTCCTGCTTGC. The relative expression level of genes was normalized to the value of GAPDH by the $2^{-\Delta \Delta \mathrm{Cq}}$ method (26), allowing the calculation of differences in gene expression using the ABI software.

Statistical analysis. CFU-F analysis, Alizarin staining, MTT and RT-qPCR analyses were repeated three times, and data are presented as the mean \pm standard deviation. Differences among the results were assessed using Bonferroni's multiple comparison tests, and statistical significance was analysed using SPSS, version 20.0 software (IBM Corp., Armonk, NY, USA). $\mathrm{P}<0.05$ was considered to indicate a statistically significant difference.

\section{Results}

EPC-CM inhibits osteogenic differentiation and promotes proliferation of BMSCs. To examine the potential influence of EPC-derived EVs on BMSCs, whether EPC-CM could regulate the osteoblastic differentiation and proliferation of BMSCs was measured. The results indicated that the OM group had an increased number of calcium deposits compared with that of the EPCs-CM + OM group (Fig. 1A). The OD ratio of both groups at day 14 also confirmed the results (Fig. 1B; $\mathrm{P}=0.003$ ). Furthermore, it was identified that the BMSCs in the EPCs-CM group formed an increased number of CFU-Fs compared with the BMSCs in culture medium (Fig. 1; $\mathrm{P}=0.0038$ ). Altogether, these results demonstrate that EPC-CM inhibits osteoblastic differentiation and 
A
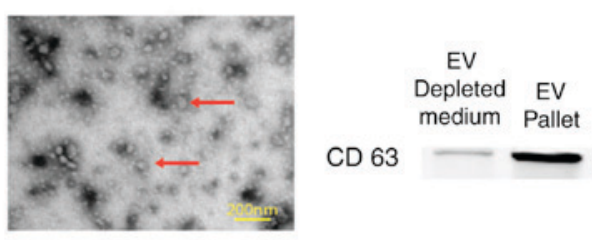

B

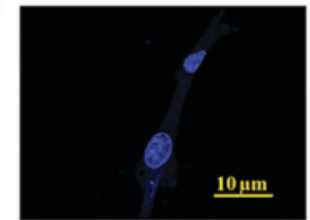

DAPI

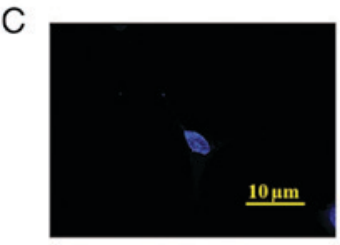

DAPI

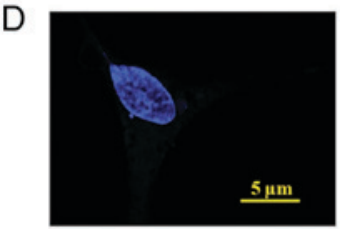

DAPI

E

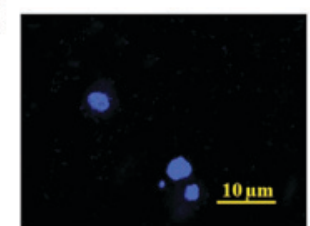

DAPI

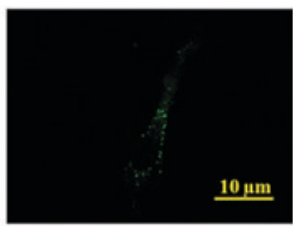

PKH67

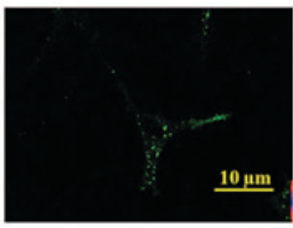

PKH67

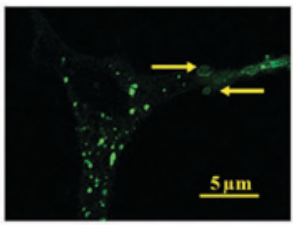

PKH67

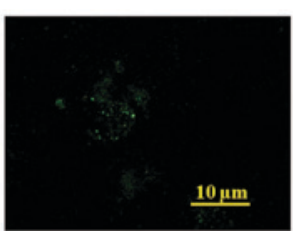

PKH67

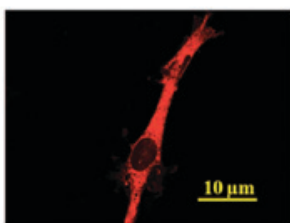

Endoplasmic reticulum

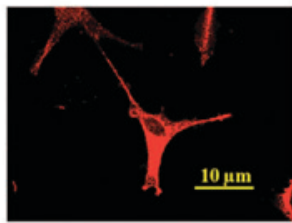

Golgi apparatus

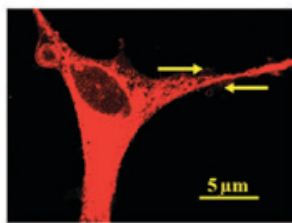

Golgi apparatus

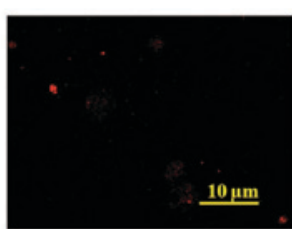

Lysosome

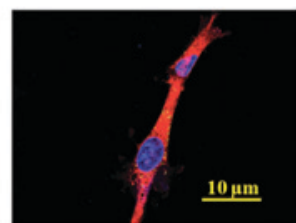

Merged

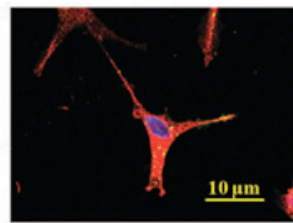

Merged

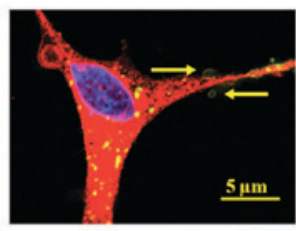

Merged

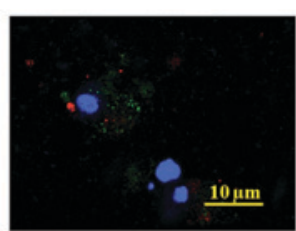

Merged

Figure 2. (A) Electron microscopic image of EPC-derived EVs (red arrows). Scale bar, $200 \mathrm{~nm}$. Western blotting of the EVs-depleted EPC conditioned medium and EV pallet also demonstrated that EPC-derived EVs were successfully isolated. (B) The EPC-derived EVs were PKH67-labelled (green fluorescence) and were added to the BMSC culture medium. Following $4 \mathrm{~h}$ of incubation, the results were analysed by confocal microscopy. The staining indicated PKH67 labelled exosomes (green) enter the cell (red, endoplasmic reticulum; blue, DAPI). (C and D) Yellow arrows indicate shallow green round-like staining suggesting that the foreign exosomes (green) were transported to and degraded by the Golgi apparatus lumen (red). (D) is a $\mathrm{x} 2$ magnification of the data presented in (C). (E) The confocal results indicate no evidence of lysosomes serving a role in exosomes degradation. EPC, endothelial progenitor cell; EV, extracellular vesicle; DAPI, 4',6-diamidino-2-phenylindole.

stimulates cell proliferation of BMSCs. These results confirm the results of an earlier report (11).

EPC-derived extracellular vesicle isolation. Prior to examining the function of EPC-derived extracellular vesicles (EPC-EVs), it was determined whether EPC-EVs are isolated and can enter BMSCs. The EPC-EVs were isolated according to a commonly used ultracentrifugation protocol and analysed using electron microscopy (Fig. 2A, red arrows) (27). In addition, CD63, a common marker of EVs, was used in western blot analysis to further confirm that EVs are purified from EPC-CM (Fig. 2A) (28). Subsequently, it was examined whether the EPC-EVs were absorbed by the BMSCs. Therefore, the collected EVs were labelled with PKH67 (green), a stable green fluorescent cell membrane linker. Subsequently, the PKH67-stained vesicles were added to the BMSC culture medium. Following a $4 \mathrm{~h}$ incubation, the cells were fixed and labelled with diverse organelle-specific dyes (red) and were imaged and analysed with confocal microscopy. As presented in Fig. 2B, the PKH67-labelled EVs (green dots) were absorbed by the BMSCs and presented in the endoplasmic reticulum (ER-Tracker Red), Golgi apparatus (Golgi-RFP) and lysosomes (Lyso-Tracker Red). These results indicated that EPC-EVs fused with the cells and were degraded by the Golgi apparatus, releasing their cargo along the way. However, it was not possible to demonstrate an association between EPC-EVs and lysosomes, which have been suggested to digest EVs (29).

EPC-derived extracellular vesicles inhibit osteogenic differentiation of BMSCs. Because EPC-EVs are absorbed by BMSCs, the function of EPC-EVs was investigated using 
A

7 days

14 days
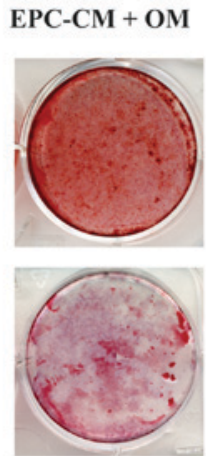

B

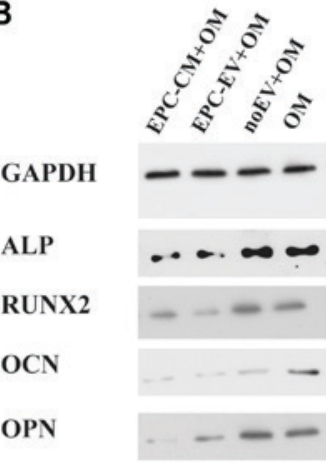

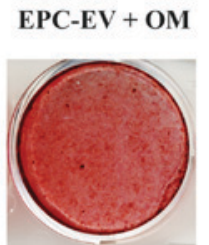

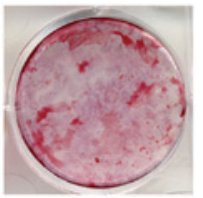

C
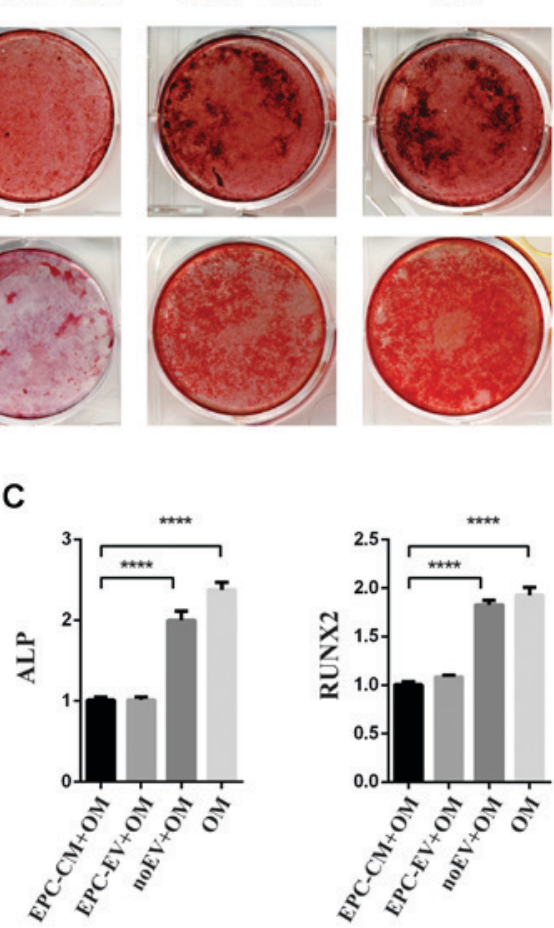
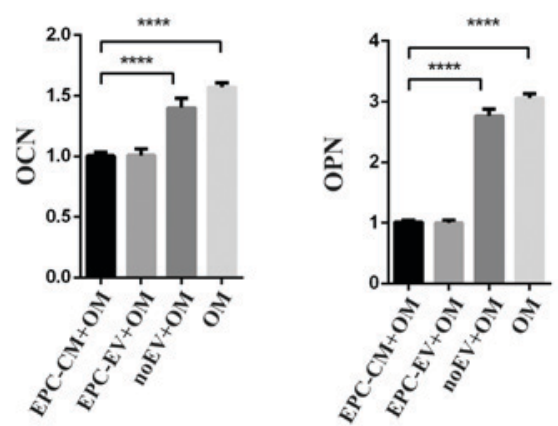

Figure 3. (A) The macroscopic Alizarin Red staining for the OM-group, the EPC-CM + OM group, EPC-EV + OM group, noEV + OM group and OM group at day 1 and day 14. (B) Western blot analysis of ALP, OCN, OPN and RUNX2 at day 14. (C) The expression of ALP, OCN, OPN and RUNX2 increased by 1.9-, 3.8-, 3.2- and 2.4-fold, respectively, in the OM group vs. the EPC-EV + OM group; ${ }^{* * * *} \mathrm{P}<0.0001$. OM, osteogenic medium; ALP, alkaline phosphatase; OCN, osteocalcin; OPN, osteopontin; RUNX2, runt-related transcription factor-2; EPC, endothelial progenitor cell; CM, conditioned medium; EV, extracellular vesicle; GAPDH, glyceraldehyde 3-phosphate dehydrogenase.

osteoblastic differentiation assays. Alizarin Red staining demonstrated that there was a marked decrease in calcium deposits in the EPC-CM + OM group and EPC-EV + OM group compared with the noEV + OM group and OM group on days 7 and 14 (Fig. 3A). Western blot analyses further confirmed that osteoblastic differentiated-related proteins were downregulated in the $\mathrm{EPC}-\mathrm{CM}+\mathrm{OM}$ group and EPC-EV + OM group (Fig. 3B). Accordingly, the expression of osteoblastic markers and osteogenic genes, including ALP, RUNX2, OPN and OCN, were additionally decreased in the $\mathrm{EPC}-\mathrm{CM}+\mathrm{OM}$ and $\mathrm{EPC}-\mathrm{EV}+\mathrm{OM}$ groups. Compared with the EV-group, the expression levels of ALP, OCN, OPN and RUNX2 were decreased by 2.4-, 2.0-, 3.1- and 1.6-fold, respectively, in the OM group (Fig. 3C). Together, these results suggested that EPC-EVs inhibited osteoblastic differentiation, presumably by modulating the expression of osteogenic genes. However, the expression of osteogenic genes in the noEV + OM group remained reduced compared with that of the OM group, thus indicating that other factors in the EPC-CM may also serve a role in regulating the osteoblastic differentiation of BMSCs.

EPC-derived extracellular vesicles regulate colony formation and proliferation of BMSCs. Given that EPC-EVs negatively regulate osteoblastic differentiation of BMSCs, it was determined whether EPC-EVs may additionally serve a role in regulating BMSCs CFU-F. Therefore, a CFU-F assay was performed and cell proliferation was analysed using MTT assays. Following 14 days of culture, CFU-Fs were increased in the EPC-CM and EPC-EV groups compared with the noEV and OM groups. Similar results were identified in the MTT assays (Fig.4). Altogether, these results suggested that EPC-derived EVs have a positive influence on the proliferation of BMSCs.

\section{Discussion}

EPCs have been demonstrated to induce vessel formation in vivo and in vitro and are commonly used in tissue 

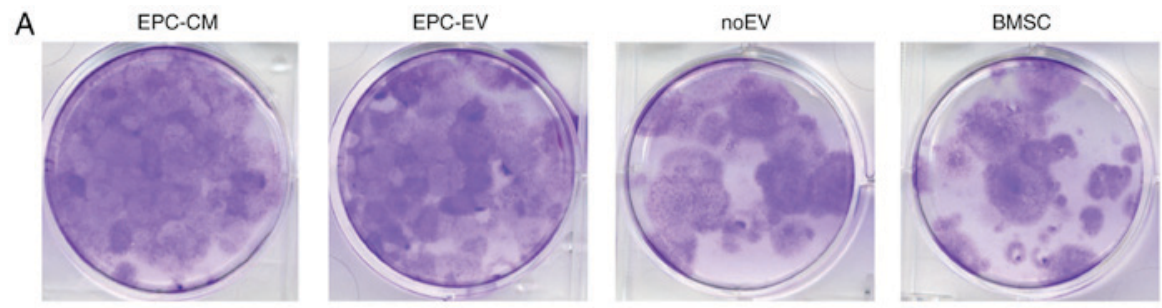

B
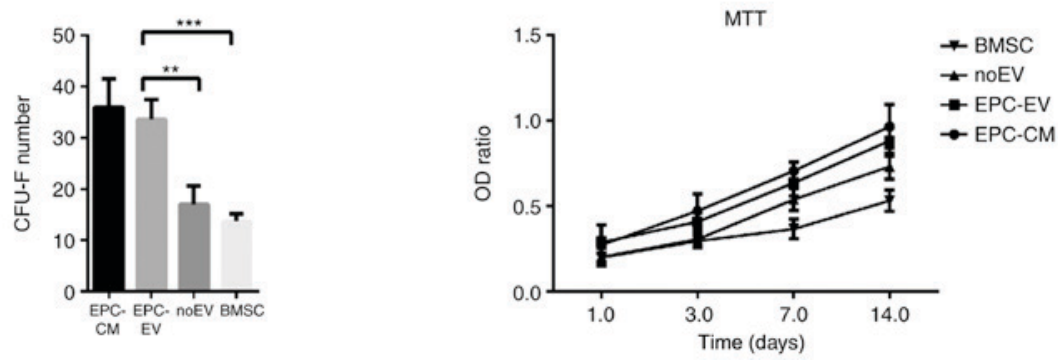

Figure 4. (A) Light microscopy image of CFU-F following 14-days of culture. (B) The CFU-F number was significantly higher in the EPC-CM (36.01 \pm 5.5 ) and the EPC-EV (33.67 \pm 3.8$)$ groups compared with the noEV (17.00 \pm 3.6$)$ and BMSC $(13.70 \pm 1.52)$ groups. There were no significant differences between the EPC-CM and the EPC-EV $(\mathrm{P}=1.00)$ groups. There was a significant difference between the EPC-EV and the noEV $(\mathrm{P}=0.005, \mathrm{P}<0.05)$ and the $\mathrm{EPC}-\mathrm{EV}$ and the BMSC $(\mathrm{P}=0.001, \mathrm{P}<0.05)$ groups. MTT analysis demonstrated the same result. ${ }^{* *} \mathrm{P}<0.01$ and ${ }^{* * * *} \mathrm{P}<0.001$. CFU-F, colony forming unit-fibroblasts; EPC, endothelial progenitor cell; CM, conditioned medium; EV, extracellular vesicle; BMSC, bone marrow stromal cell.

engineering to promote bone regeneration (30). EPCs exhibit good vessel formation when coated with matrigel (31) and attenuate limb ischaemic mouse models (32). However, EPCs have a controversial influence on the proliferation and osteoblastic differentiation of BMSCs. Duttenhoefer et al (11) demonstrated that BMSCs co-cultured with EPCs exhibit a significantly reduced level of osteogenic gene expression and less calcium deposition. Furthermore, Wen et al (33) established an EPC/BMSC indirect Transwell culture system, and identified that co-cultured BMCs proliferate more than mono-cultured MSCs. In the present study, the osteoblastic differentiation of BMSCs treated with EPC-derived EVs was inhibited, whereas the number of CFU-Fs was increased.

Although the osteoblastic differentiation of BMSCs is inhibited by EPC-EVs, the exact mechanism remains unclear. EVs deliver a large variety of cargo, including proteins, DNA and RNA. The proteins and RNA in EVs serve critical roles in regulating the differentiation and function of recipient cells (34). Spectrometry data of EVs have identified over 4,000 different proteins in the exosomes (35). Although the proteins differ substantially according to their origin and have different functions, some proteins are shared by all types of EVs (36). These shared proteins are associated with cell-to-cell communication. Trafficking-associated proteins, including heat shock protein (HSP) 70 and HSP90, and cytoskeletal proteins, including myosin, actin and tubulin, are widely distributed in EVs (37). Additionally, studies have demonstrated that small RNAs in EVs also have an influence on target cells $(38,39)$. Koppers-Lalic et al (34) reviewed the known small RNAs in EVs and noted that the functional RNAs are critical in the regulation of cell commitment, differentiation and activity. Therefore, profiling of proteins, DNAs and RNAs in EPC-derived EVs will aid in the exploration of communication between EPCs and BMSCs.

The confocal microscopy data indicated that the green round-like fluorescence (red arrow) in the EVs was internalized, transported to and degraded by the Golgi apparatus, the lumen of which was stained red (white arrow). These results suggested that the cargo in the EVs was released in the Golgi apparatus rather than in the lysosomes. However, the exact mechanism of EV degradation remains controversial. Baixauli et al (40) observed that EVs that are internalized by cells may fuse with either the lysosomes or the multivesicular bodies. Alvarez-Erviti et al (41) proposed that lysosomal dysfunction in SH-SY5Y cells is associated with increased EV release, thus indicating a critical role of lysosomes in EV degradation. Previous studies have demonstrated that other cell organelles may also serve a prominent role in EV degradation. Campanella et al (42) identified that EVs are transported to the Golgi apparatus by HSP60 located in tumor cells. A previous study additionally demonstrated that the Golgi apparatus is key to EV degradation (43). Therefore, how the cells degrade the EVs requires further investigation.

In conclusion, EPC-CM inhibits osteogenic gene expression and calcium deposition in BMSCs in vitro. EPC-derived EVs are one of the major regulators in EPC-CM. Osteoblastic differentiation of BMSCs was inhibited by EPC-derived EVs, whereas BMSC proliferation was increased. BMSCs treated with EPC-derived EVs exhibited fewer calcium deposits however increased CFU-Fs. Western blot analysis and RT-qPCR results also demonstrated that osteoblastic differentiation was inhibited. In addition, it was observed that EPC-EVs were delivered to and degraded by the Golgi apparatus rather than by the lysosomes in BMSCs. The present study demonstrated that EPCs communicate and regulate BMSCs through EPC-derived EVs and provides a foundation for further exploration of the communication between EPCs and BMSCs.

\section{Acknowledgements}

The current study was funded by the National Natural Science Foundation of China (grant no. 81272003 to Dr Changqing Zhang). 


\section{References}

1. Dhahri D, Sato-Kusubata K, Ohki-Koizumi M, Nishida C, Tashiro Y, Munakata S, Shimazu H, Salama Y, Eiamboonsert S, Nakauchi $\mathrm{H}$, et al: Fibrinolytic crosstalk with endothelial cells expands murine mesenchymal stromal cells. Blood 128: 1063-1075, 2016.

2. Bradley EW, Carpio LR, van Wijnen AJ, McGee-Lawrence ME and Westendorf JJ: Histone deacetylases in bone development and skeletal disorders. Physiol Rev 95: 1359-1381, 2015.

3. Seeman E: Bone modeling and remodeling. Crit Rev Eukaryot Gene Expr 19: 219-233, 2009.

4. Ornitz DM and Marie PJ: FGF signaling pathways in endochondral and intramembranous bone development and human genetic disease. Genes Dev 16: 1446-1465, 2002.

5. Kronenberg HM: Developmental regulation of the growth plate. Nature 423: 332-336, 2003.

6. Kanczler JM and Oreffo RO: Osteogenesis and angiogenesis: The potential for engineering bone. Eur Cell Mater 15: 100-114, 2008.

7. Almubarak S, Nethercott H, Freeberg M, Beaudon C, Jha A, Jackson W, Marcucio R, Miclau T, Healy K and Bahney C: Tissue engineering strategies for promoting vascularized bone regeneration. Bone 83: 197-209, 2016.

8. Asahara T, Murohara T, Sullivan A, Silver M, van der Zee R, Li T, Witzenbichler B, Schatteman G and Isner JM: Isolation of putative progenitor endothelial cells for angiogenesis. Science 275: 964-967, 1997.

9. De Palma M, Murdoch C, Venneri MA, Naldini L and Lewis CE: Tie2-expressing monocytes: Regulation of tumor angiogenesis and therapeutic implications. Trends Immunol 28: 519-524, 2007.

10. van der Pouw Kraan TC, van der Laan AM, Piek JJ and Horrevoets AJ: Surfing the data tsunami, a bioinformatic dissection of the proangiogenic monocyte. Vascul Pharmacol 56: 297-305, 2012

11. Duttenhoefer F, de Freitas RL, Loibl M, Bittermann G, Richards RG, Alini M and Verrier S: Endothelial progenitor cel fraction contained in bone marrow-derived mesenchymal stem cell populations impairs osteogenic differentiation. Biomed Res Int 2015: 659542, 2015.

12. Goerke SM, Obermeyer J, Plaha J, Stark GB and Finkenzeller G: Endothelial progenitor cells from peripheral blood support bone regeneration by provoking an angiogenic response. Microvasc Res 98: 40-47, 2015.

13. Raposo G and Stoorvogel W: Extracellular vesicles: Exosomes, microvesicles, and friends. J Cell Biol 200: 373-383, 2013.

14. Pan BT and Johnstone RM: Fate of the transferrin receptor during maturation of sheep reticulocytes in vitro: Selective externalization of the receptor. Cell 33: 967-978, 1983.

15. Thery C, Regnault A, Garin J, Wolfers J, Zitvogel L, Ricciardi-Castagnoli P, Raposo G and Amigorena S: Molecular characterization of dendritic cell-derived exosomes. Selective accumulation of the heat shock protein hsc73. J Cell Biol 147: 599-610, 1999

16. Raposo G, Nijman HW, Stoorvogel W, Liejendekker R, Harding CV, Melief CJ and Geuze HJ: B lymphocytes secrete antigen-presenting vesicles. J Exp Med 183: 1161-1172, 1996.

17. Mears R, Craven RA, Hanrahan S, Totty N, Upton C, Young SL, Patel P, Selby PJ and Banks RE: Proteomic analysis of melanoma-derived exosomes by two-dimensional polyacrylamide gel electrophoresis and mass spectrometry. Proteomics 4: 4019-4031, 2004.

18. Raposo G, Tenza D, Mecheri S, Peronet R, Bonnerot C and Desaymard C: Accumulation of major histocompatibility complex class II molecules in mast cell secretory granules and their release upon degranulation. Mol Biol Cell 8: 2631-2645, 1997.

19. Blanchard N, Lankar D, Faure F, Regnault A, Dumont C, Raposo G and Hivroz C: TCR activation of human T cells induces the production of exosomes bearing the TCR/CD3/zeta complex. J Immunol 168: 3235-3241, 2002.

20. van Niel G, Raposo G, Candalh C, Boussac M, Hershberg R, Cerf-Bensussan $\mathrm{N}$ and Heyman M: Intestinal epithelial cells secrete exosome-like vesicles. Gastroenterology 121: 337-349, 2001.

21. Burger D, Viñas JL, Akbari S, Dehak H, Knoll W, Gutsol A, Carter A, Touyz RM, Allan DS and Burns KD: Human endothelial colony-forming cells protect against acute kidney injury: Role of exosomes. Am J Pathol 185: 2309-2323, 2015.
22. Denzer K, van Eijk M, Kleijmeer MJ, Jakobson E, de Groot C and Geuze HJ: Follicular dendritic cells carry MHC class II-expressing microvesicles at their surface. J Immunol 165: $1259-1265,2000$

23. Morelli AE, Larregina AT, Shufesky WJ, Sullivan ML, Stolz DB, Papworth GD, Zahorchak AF, Logar AJ, Wang Z, Watkins SC, et al: Endocytosis, intracellular sorting, and processing of exosomes by dendritic cells. Blood 104: 3257-3266, 2004.

24. Clayton A, Turkes A, Dewitt S, Steadman R, Mason MD and Hallett MB: Adhesion and signaling by B cell-derived exosomes: The role of integrins. FASEB J 18: 977-979, 2004.

25. Meirelles Lda S and Nardi NB: Murine marrow-derived mesenchymal stem cell: Isolation, in vitro expansion, and characterization. Br J Haematol 123: 702-711, 2003.

26. Livak KJ and Schmittgen TD: Analysis of relative gene expression data using real-time quantitative PCR and the 2(-Delta Delta C(T)) method. Methods 25: 402-408, 2001.

27. Lobb RJ, Becker M, Wen SW, Wong CS, Wiegmans AP, Leimgruber A and Möller A: Optimized exosome isolation protocol for cell culture supernatant and human plasma. J Extracell Vesicles 4: 27031, 2015.

28. Narayanan R, Huang CC and Ravindran S: Hijacking the Cellular Mail: Exosome mediated differentiation of mesenchymal stem cells. Stem Cells Int 2016: 3808674, 2016.

29. Fleury A, Martinez MC and Le Lay S: Extracellular vesicles as therapeutic tools in cardiovascular diseases. Front Immunol 5: 370, 2014.

30. Sivaraj KK and Adams RH: Blood vessel formation and function in bone. Development 143: 2706-2715, 2016.

31. Gulati R, Jevremovic D, Peterson TE, Chatterjee S, Shah V, Vile RG and Simari RD: Diverse origin and function of cells with endothelial phenotype obtained from adult human blood. Circ Res 93: 1023-1025, 2003.

32. Yamaguchi J, Kusano KF, Masuo O, Kawamoto A, Silver M, Murasawa S, Bosch-Marce M, Masuda H, Losordo DW, Isner JM and Asahara T: Stromal cell-derived factor-1 effects on ex vivo expanded endothelial progenitor cell recruitment for ischemic neovascularization. Circulation 107: 1322-1328, 2003.

33. Wen L, Wang Y, Wen N, Yuan G, Wen M, Zhang L, Liu Q, Liang Y, Cai C, Chen X and Ding Y: Role of endothelial progenitor cells in maintaining stemness and enhancing differentiation of mesenchymal stem cells by indirect cell-cell interaction. Stem Cells Dev 25: 123-138, 2016.

34. Koppers-Lalic D, Hogenboom MM, Middeldorp JM and Pegtel DM: Virus-modified exosomes for targeted RNA delivery; a new approach in nanomedicine. Adv Drug Deliv Rev 65: 348-356, 2013.

35. Mathivanan S, Ji H and Simpson RJ: Exosomes: Extracellular organelles important in intercellular communication. J Proteomics 73: 1907-1920, 2010.

36. Simpson RJ, Jensen SS and Lim JW: Proteomic profiling of exosomes: Current perspectives. Proteomics 8: 4083-4099, 2008.

37. van Dommelen SM, Vader P, Lakhal S, Kooijmans SA, van Solinge WW, Wood MJ and Schiffelers RM: Microvesicles and exosomes: Opportunities for cell-derived membrane vesicles in drug delivery. J Control Release 161: 635-644, 2012.

38. Gibbings D and Voinnet O: Control of RNA silencing and localization by endolysosomes. Trends Cell Biol 20: 491-501, 2010.

39. Pegtel DM, Cosmopoulos K, Thorley-Lawson DA, van Eijndhoven MA, Hopmans ES, Lindenberg JL, de Gruijl TD, Würdinger T and Middeldorp JM: Functional delivery of viral miRNAs via exosomes. Proc Natl Acad Sci USA 107: 6328-6333, 2010.

40. Baixauli F, Lopez-Otin C and Mittelbrunn M: Exosomes and autophagy: Coordinated mechanisms for the maintenance of cellular fitness. Front Immunol 5: 403, 2014.

41. Alvarez-Erviti L, Seow Y, Schapira AH, Gardiner C, Sargent IL, Wood MJ and Cooper JM: Lysosomal dysfunction increases exosome-mediated alpha-synuclein release and transmission. Neurobiol Dis 42: 360-367, 2011.

42. Campanella C, Bucchieri F, Merendino AM, Fucarino A, Burgio G, Corona DF, Barbieri G, David S, Farina F, Zummo G, et al: The odyssey of Hsp60 from tumor cells to other destinations includes plasma membrane-associated stages and Golgi and exosomal protein-trafficking modalities. PLoS One 7: e42008, 2012.

43. Qin Y, Wang L, Gao Z, Chen G and Zhang C: Bone marrow stromal/stem cell-derived extracellular vesicles regulate osteoblast activity and differentiation in vitro and promote bone regeneration in vivo. Sci Rep 6: 21961, 2016. 